PATIENT SAFETY

C.B. Nauer

A. Rieke

C. Zubler

C. Candreia

A. Arnold

P. Senn

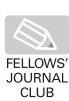

\title{
Low-Dose Temporal Bone CT in Infants and Young Children: Effective Dose and Image Quality
}

BACKGROUND AND PURPOSE: The temporal bone is ideal for low-dose CT because of its intrinsic high contrast. The aim of this study was to retrospectively evaluate image quality and radiation doses of a new low-dose versus a standard high-dose pediatric temporal bone CT protocol and to review dosimetric data from the literature.

MATERIALS AND METHODS: Image quality and radiation doses were compared for 38 low-dose (80 kV/90-110 mAs) and 16 high-dose (140 kV/170 mAs) temporal bone CT scans of infants to 5-year-old children. The CT visualization quality of 23 middle and inner ear structures was subjectively graded by 3 neuroradiologists and 3 otologists by using a 5-point scale with scores 1-2 indicating insufficient and scores 3-5 indicating sufficient image quality. Effective doses of local and literature-derived protocols were calculated from dosimetric data by using NRPB-SR250 software.

RESULTS: Insufficient image-quality scores were more frequent in low-dose scans versus high-dose scans, but the difference was only statistically significant for otologists $(6.0 \%$ versus $3.4 \%, P=.004)$ and not for neuroradiologists $(1.2 \%$ versus $0.7 \%, P=.84)$. Image quality was critical for small structures (such as the stapes or lamella at the internal auditory canal fundus). Effective doses were 0.25-0.3 mSv for low-dose scans, 1.4-1.8 mSv for high-dose scans, and 0.9-2.6 mSv for literaturederived protocols.

CONCLUSIONS: The image quality of the new low-dose protocol remains diagnostic for assessing middle and inner ear anatomy despite a 3- to 8-fold dose reduction over previous and literature-derived protocols. However, image quality of small structures is critical and may be perceived as insufficient.

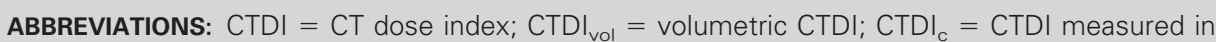
the center; $C T D I_{p}=C T D I$ measured in the periphery; $C T D I_{w}=$ weighted $C T D I ; D_{\text {eff }}=$ effective dose; $\mathrm{DLP}=$ dose-length product

$\mathbf{T}$ he use of adult settings in pediatric CT leads to unnecessarily high radiation doses, and the current literature recommends using adapted settings for pediatric CT. ${ }^{1,2}$ As a result, the National Cancer Institute guidelines ${ }^{1}$ on pediatric CT call for the development of specific pediatric CT protocols as a long-term strategy for reducing radiation exposure. As part of a local dose-optimization program, all CT protocols were revised at our institution in 2006 and 2007. When the low-dose protocols were created, an extensive literature search was performed first. Then, beginning at an established image-quality level, dose-relevant scanning parameters were reduced stepwise, as long as the image quality remained diagnostic, as judged in consensus by neuroradiologists and the involved clinicians.

For our dose-optimization program and this study, we defined the term "low-dose protocol" as one that is optimized for the lowest feasible radiation exposure that maintains a basic yet diagnostic image quality while a substantial dose reduction is reached compared with a standard-dose CT that offers a good-to-excellent image quality. ${ }^{3}$

Received September 3, 2010; accepted after revision December 22.

From the Institute for Diagnostic and Interventional Neuroradiology (C.B.N., A.R., C.Z.) and University Department of ENT, Head and Neck Surgery (C.C., A.A., P.S.), University of Berne, Berne, Switzerland.

Paper previously presented in part at: Annual Congress of the European Society of Neuroradiology, September 19-23, 2009; Athens, Greece.

Please address correspondence to Andreas Arnold, MD, University Department of ENT, Head and Neck Surgery, Inselspital, University of Berne, Freiburgstra 4, CH-3010 Berne, Switzerland; e-mail: andreas.arnold@insel.ch.

http://dx.doi.org/10.3174/ajnr.A2524
High-resolution temporal bone CT in young pediatric patients is, at our institution, mainly used for the preoperative assessment of the anatomy in cochlear implant candidates but also for diagnosing ossicular dysplasia, other dysplasias, and fractures. For high-resolution CT of the temporal bone, a helical scan is generally performed with submillimeter sections. Then, images are reconstructed with a small FOV and an edgeenhancing kernel to obtain the highest possible spatial resolution in all 3 dimensions. We use an FOV of $100 \mathrm{~mm}$, and $0.625-\mathrm{mm}$ sections are reconstructed every $0.2 \mathrm{~mm}$. In our current low-dose protocol, $80 \mathrm{kV}$ and $90-110 \mathrm{mAs}$ are used for children younger than 1 year and those $1-5$ years of age. The resulting dataset allows the visualization of temporal bone anatomy in arbitrary planes by using multiplanar reformations.

The temporal bone is ideally suited for low-dose CT because of the high intrinsic contrast of the imaged structures. ${ }^{4}$ As a consequence, low-dose temporal bone CT protocols for adults have been described before. ${ }^{4,5}$ However, there is no systematic evaluation of any pediatric low-dose temporal bone CT protocol in the literature. The purposes of this study were, therefore, to systematically assess whether the image quality of our low-dose temporal bone CT protocol is sufficient for diagnostic purposes, to calculate the effective radiation doses of low-dose and high-dose protocols, and to compare them with literature-derived data.

Today, scans are often reviewed by the clinicians, especially if they intend to operate on the patient; we think that scanning protocols should be implemented in collaboration with the involved clinicians. Therefore, our low-dose protocol for tem- 
poral bone imaging was elaborated and evaluated (in the present study) in collaboration with otologists.

\section{Materials and Methods}

This retrospective study was performed in full accordance with regulations issued by the local ethics committee.

\section{Patient Selection Criteria}

All patients younger than 5 years of age undergoing CT of the temporal bone since the introduction of the low-dose protocol were retrospectively recruited from our PACS. Only scans showing normal anatomic inner and middle ear structures (as in the written record) were included; scans showing motion artifacts were not included.

Scans showing isolated dysplasia of the external ear and other findings not affecting middle and inner ear anatomy were included. Scans showing obvious pathologies like middle/inner ear dysplasia or infectious conditions were excluded. The indications for imaging were the following: congenital hearing impairment/cochlear implant evaluation $(n=18)$, trauma $(n=3)$, infection $(n=2)$, external auditory canal atresia $(n=2)$, histiocytosis $(n=1)$, and middle ear dysplasia $(n=1)$.

The electronic archive was searched for scans to use as a standard of reference from November 2003 (introduction of electronic archive) to March 2007 (introduction of age-specific protocols). Due to the lack of standardized pediatric protocols before the introduction of the low-dose protocol, the dose-relevant scan parameters were chosen by the attending neuroradiologist at that time. To obtain a homogeneous set of high-dose reference scans, we included only examinations performed with the highest dose in the high-dose group for this study.

\section{Patient Characteristics}

A total of 38 temporal bone studies of 19 pediatric patients were included in the low-dose group. Eight patients (16 scans) belonged to the age group younger than 1 year (mean age, 9 months; range, 7-10 months), and 11 patients (22 scans) belonged to the age group of 1-5 years (mean age, 30 months; range, 17-59 months). A total of 16 temporal bone studies of 8 pediatric patients were included in the high-dose group. One child (2 scans) belonged to the age group younger than 1 year ( 8 months), and 7 children belonged to the age group 1-5 years (mean age, 25 months; range, 13-52 months).

\section{Review of Scans}

Each study was independently analyzed by 3 neuroradiologists (C.B.N., A.R., C.Z.) and 3 otologists (C.C., A.A., P.S.); each investigator had at least 5 years of experience in reviewing temporal bone $C T$ scans (C.B.N., 7 years; A.R., 6 years; C.Z., 6 years; C.C., 5 years; A.A., 8 years; P.S., 11 years). Image analysis was performed on an EasyVision picture-viewing station (Philips Healthcare, Best, the Netherlands) and included axial sections and multiplanar reformations generated with the integrated software tool. In accordance with our clinical routine, reviewers were free to choose the appropriate planes and window width/center level settings. The optimal window/level settings to review the images were recorded for each temporal bone. Readers were blinded to the dose-relevant scan parameters during the review of the scan.

Twenty-three normal anatomic structures (Table 1) were assessed with regard to image quality by using a rating system from 5 to 1 in descending order: 5 , very good delineation of structure and excellent image quality; 4 , clear delineation of structure and good image qual-
Table 1: Synopsis of the 23 anatomic structures reviewed and the respective primary criteria for image quality assessment

\begin{tabular}{|c|c|}
\hline Structure/Condition & Review Criteria \\
\hline Cochlea & Normal contour, 2.5 turns \\
\hline Cochlear patency & $\begin{array}{l}\text { Ability to discern intracochlear } \\
\text { ossifications }\end{array}$ \\
\hline Spiral osseous lamina & Presence, integrity \\
\hline Modiolus & Presence, integrity \\
\hline Labyrinth & Contour, density \\
\hline Vestibular aqueduct & Contour, density \\
\hline Cochlear aqueduct & Contour, density \\
\hline Cochlear nerve canal & Presence \\
\hline $\begin{array}{l}\text { Bony lamella at auditory } \\
\text { canal fundus }\end{array}$ & $\begin{array}{l}\text { Presence, integrity of bony lamella } \\
\text { separating the internal } \\
\text { auditory canal from the cochlea }\end{array}$ \\
\hline Internal auditory canal & Contour \\
\hline $\begin{array}{l}\text { Facial nerve canal, cochlear } \\
\text { segment }\end{array}$ & Contour, course \\
\hline $\begin{array}{l}\text { Facial nerve canal, tympanic } \\
\text { segment }\end{array}$ & Contour, course \\
\hline $\begin{array}{l}\text { Facial nerve canal, mastoid } \\
\text { segment }\end{array}$ & Contour, course \\
\hline Middle ear cavity & Aeration \\
\hline Malleus & Presence of all parts \\
\hline Incus & Presence of all parts \\
\hline Stapes & Presence of all parts \\
\hline Round window & Presence, aperture \\
\hline Round window niche & Borders, aeration \\
\hline Oval window & Presence, borders, footplate position \\
\hline Internal carotid artery canal & Borders, osseous wall dehiscence \\
\hline Jugular foramen & Borders, osseous wall dehiscence \\
\hline Mastoid & Bony borders, aeration \\
\hline
\end{tabular}

ity; 3, anatomic structures still fully assessable in all parts and acceptable image quality; 2, structures identifiable, but no details assessable, resulting in insufficient image quality; and 1, anatomic structures not identifiable due to poor image quality.

For the image-quality assessment, we chose the anatomic structures that were the most susceptible to image-quality degradation with increasing image noise (such as the stapes and the cochlear spiral osseous lamina) and that were also important for patients with congenital hearing deficits and/or are cochlear implant candidates (such as the internal structures of the cochlea and the facial nerve) on the basis of our experiences.

\section{Image Acquisition Technique}

All scans were acquired in spiral mode with an 8-section CT scanner (LightSpeed Ultra; GE Healthcare, Milwaukee, Wisconsin). We used the following parameters: collimation, $0.625 \mathrm{~mm}$; pitch, 1 ; FOV, 100 $\mathrm{mm}$. Sections of $0.625-\mathrm{mm}$ thickness were reconstructed independently for each side with an overlap of $68 \%$. An edge-enhancing reconstruction kernel (Bone Plus; GE Healthcare) was used. Imageacquisition parameters for the low-dose and high-dose scans were identical except for the tube voltage and tube current. For infants younger than 1 year, $80 \mathrm{kV}$ and $90 \mathrm{mAs}$ were chosen, which correspond to a CTDI ${ }_{\mathrm{vol}}$ of $8.8 \mathrm{mGy}$ (as calculated by the scanner software). For children between 1 and 5 years of age, $80 \mathrm{kV}$ and $110 \mathrm{mAs}$ $\left(\mathrm{CTDI}_{\mathrm{vol}}=10.8 \mathrm{mGy}\right)$ were chosen; settings used for the high-dose scans were $140 \mathrm{kV}$ and $170 \mathrm{mAs}\left(\mathrm{CTDI}_{\mathrm{vol}}=63 \mathrm{mGy}\right)$. Generally, the temporal bone was imaged from the tip of the mastoid process to the tegmen tympani. 


\section{Dosimetry}

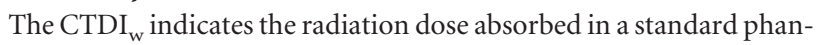
tom during axial scanning. It is obtained by measuring the radiationdose profile along a $100-\mathrm{mm}$ range of the $\mathrm{z}$-axis in the center $\left(\mathrm{CTDI}_{\mathrm{c}}\right.$ ) and the periphery $\left(\mathrm{CTDI}_{\mathrm{p}}\right)$ of the phantom. Then, the $\mathrm{CTDI}_{\mathrm{w}}$ is calculated as $\mathrm{CTDI}_{\mathrm{w}}=1 / 3 \mathrm{CTDI}_{\mathrm{c}}+2 / 3 \mathrm{CTDI}_{\mathrm{p}}$. For helical scanning, the $\mathrm{CTDI}_{\mathrm{vol}}$ is defined as $\mathrm{CTDI}_{\mathrm{vol}}=\mathrm{CTDI} /$ pitch. The CTDI is measured in milligrays. ${ }^{6}$

The CTDI is independent of the scan length. As a dosimetric unit expressing the dose absorbed over the whole scan length, the DLP is defined as DLP $=\mathrm{CTDI}_{\mathrm{vol}} \times$ scan length. Its unit is milligray centimeter. To obtain a measure of the stochastic risk resulting from ionizing radiation, the effective dose was used, which is the sum of all organ doses of the body corrected for a tissue-weighting factor for each organ. Its unit is millisievert. ${ }^{6}$

The CTDI ${ }_{\mathrm{vol}}$ and the DLP are displayed on the scanner console after the examination and are stored in a file with the scans. The DLPs of all examinations included in this study were collected retrospectively. The mean DLPs and mean scan lengths (younger than 1 year, $4.3 \mathrm{~cm} ; 1-5$ years, $4.6 \mathrm{~cm}$ ) were used to calculate the effective doses for a 1-year-old infant and a 5 -year old child by using the Monte Carlo simulation software NRPB-SR250. ${ }^{7}$

A literature search was performed to find dosimetric data from protocols described or recommended in the literature. Where appropriate and feasible, effective doses were calculated. The calculations were made by using the scan lengths of our cases (see above). Modern 16- to 64-section scanner models from the 4 major CT manufacturers were assumed for these calculations.

\section{Statistical Analysis}

Image-quality score frequencies were calculated for each reviewer group. The absolute frequencies (for example, the number of times the score 4 was given by the neuroradiologists for the stapes) were not directly comparable between the low- and the high-dose scans because the case numbers were not equal. Therefore, the relative frequencies were calculated. The relative score frequencies of all anatomic structures were tested pair-wise for statistically significant differences between the low- and the high-dose scans by using the Wilcoxon signed rank test,. A $P<.05$ was considered significant (Bonferroni-corrected for multiple comparisons).

The difference between scores 5 and 4 is less important to the clinical usefulness of a scan than the difference between the scores 3 and 2. Thus, the data were additionally dichotomized into 2 clinically relevant categories: "sufficient image quality" (scores 5, 4, and 3) versus "insufficient image quality" (scores 2 and 1). The relative frequencies of insufficient quality images were tested for statistically significant differences between the low- and the high-dose scans by using the Wilcoxon signed rank test; a $P<.05$ was considered significant. Furthermore, the interobserver agreement (free-margin multirater $\kappa$ ) for the reviewer groups was calculated for these 2 clinically relevant categories.

The window/level settings used for the low- and the high-dose scans were tested for statistically significant differences with the Mann-Whitney $U$ test with a 2 -tailed $P$ value; a $P<.05$ was considered significant.

\section{Results}

\section{Image Quality Assessment}

The mean frequencies of image quality scores for low- and high-dose scans are shown in Fig 1.
In the neuroradiologist group (Fig $1 A$ ), the frequency of score 5 was significantly lower for the low-dose scans versus high-dose scans (30.1\% versus $46.1 \%$, respectively, $P<.001$ ), whereas the frequency of scores $4(51.8 \%$ versus $42.4 \%$, respectively, $P<.015)$ and $3(10.8 \%$ versus $16.9 \%$, respectively, $P<.003)$ was significantly higher for low-dose scans. The frequency of the scores 1 and 2 was very low for both protocols $(<1.5 \%)$, and the difference did not reach statistical significance. Similarly, when pooling the scores 1 and 2 into 1 group of insufficient scores, the difference between low- and highdose scans was still not statistically significant $(1.2 \%$ versus $0.7 \%$, respectively, $P=.84$, Fig $1 C$ )

Only 2 structures had $>5 \%$ insufficient ratings in the lowdose scans when reviewed by neuroradiologists: the stapes (12.3\%, $n=14 / 114$ ratings) and the internal auditory canal fundus $(7 \%, 8 / 114)$. In general, a high rate of insufficient ratings strongly correlated with low interobserver agreement (Spearman rank correlation coefficient, $r=-0.998$ ). For example, of 8 insufficient ratings for the internal auditory canal fundus, each sample received only 1 insufficient rating, and these ratings were given by a single person, while the other 2 reviewers considered the image quality to be sufficient. Thus, despite $>5 \%$ insufficient ratings for some structures, the anatomy was in fact sufficiently visible in most cases by most reviewers. All other structures had $<5 \%$ insufficient ratings.

Among the otologists (Fig $1 B$ ), score 1 alone was not significantly more frequent in low-dose scans versus high-dose scans $(0.8 \%$ versus $0.5 \%$, respectively, $P>.15)$. In contrast, scores $2(5.2 \%$ versus $3.0 \%$, respectively, $P<.004)$ and 3 (53.9\% versus $20.7 \%$, respectively, $P<.002$ ) were significantly more frequent in low-dose scans. The highest scores $(4$ and 5) were given less frequently in low-dose compared with high-dose scans $(24.4 \%$ versus $61.1 \%$, respectively, $P<.0001$; and $13.4 \%$ versus $14.5 \%$, respectively, $P>.34$ ), though the difference was only statistically significant for the score 4 . When we pooled scores 1 and 2 into a group of insufficient scores, the frequency attributed to the insufficient group was significantly higher for low-dose scans versus high-dose scans (6.0\% versus $3.4 \%$, respectively, $P<.004$; Fig $1 C$ ).

Eight structures/conditions had $>5 \%$ insufficient ratings in the low-dose scans reviewed by otologists: cochlear patency (7\%, $n=8 / 114$ ratings), the vestibular aqueduct (16.7\%), the cochlear aqueduct $(20.2 \%)$, the bony lamella at the internal auditory canal fundus $(13.2 \%)$, the mastoid segment of the facial nerve $(14.9 \%)$, the stapes $(28.1 \%)$, the round window (7.9\%), and the oval window (11.4\%). Six structures had $>5 \%$ insufficient ratings in high-dose scans reviewed by otologists: modiolus $(6.3 \%, 3 / 48)$, the vestibular aqueduct $(14.6 \%)$, the cochlear aqueduct $(12.5 \%)$, the mastoidal segment of the facial nerve $(10.4 \%)$, the stapes $(12.5 \%)$, and the oval window (6.25\%).

The interobserver agreement $\kappa$ for neuroradiologists was very high, with a mean for all structures of 0.96 (range, 0.721.0) for low-dose and 0.97 (range, 0.83-1.0) for high-dose scans. For otologists, the interobserver agreement $\kappa$ was lower, with a mean of 0.82 (range, 0.5-1.0) for low-dose and 0.88 (range, 0.5-1.0) for high-dose scans.

The mean window/level settings ( $\pm 1 \mathrm{SD})$ used by neuroradiologists were $1212 \pm 391 \mathrm{HU}$ and $6136 \pm 1552 \mathrm{HU}$ for low-dose and $854 \pm 348 \mathrm{HU}$ and $4265 \pm 1118 \mathrm{HU}$ for high- 

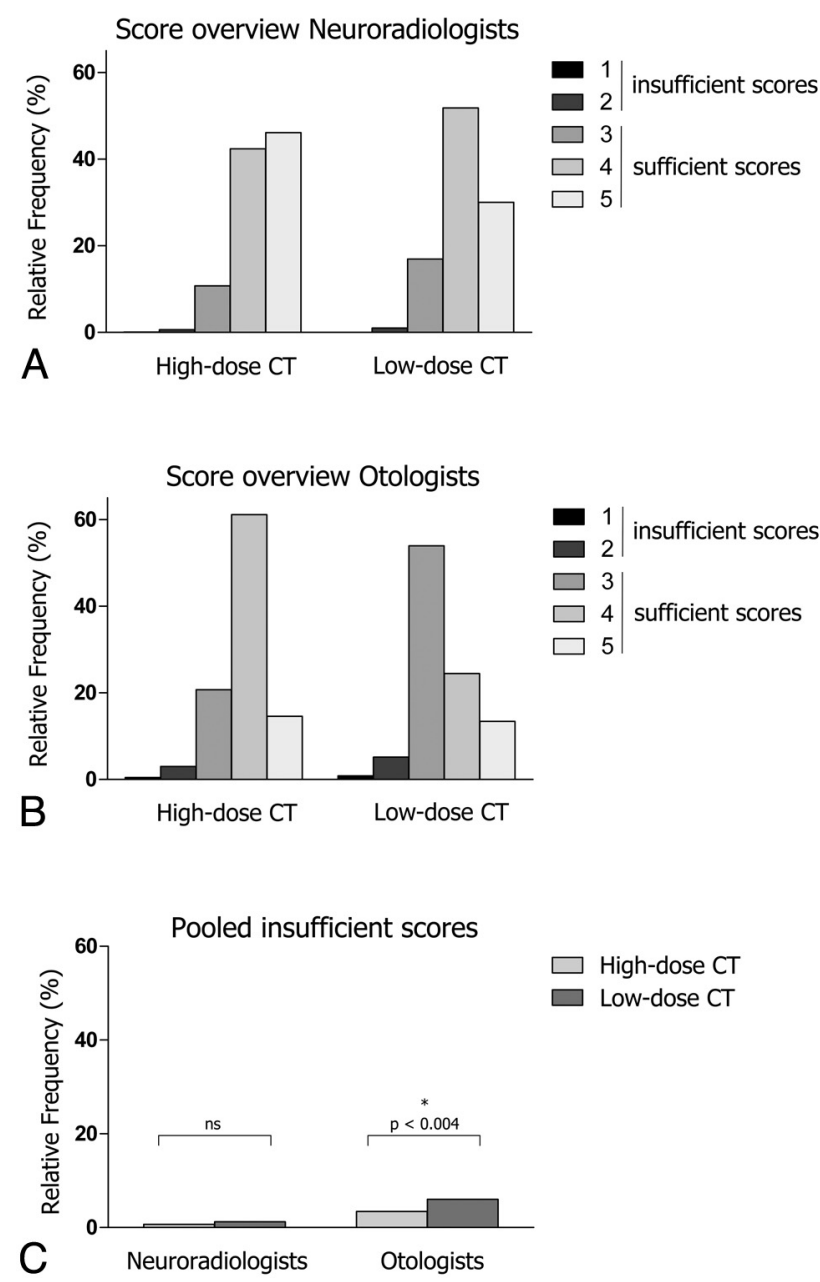

Fig 1. $A$ and $B$, The relative frequencies of scores $1-5$ are shown for the neuroradiologists $(A)$ and the otologists $(B)$ and for high-dose and low-dose CT. C. The pooled insufficient scores are shown for the neuroradiologists and the otologists for high-dose versus low-dose CT.

dose scans. The mean window/level settings used by otologists were $1003 \pm 508 \mathrm{HU}$ and $4396 \pm 1933 \mathrm{HU}$ for low-dose and $656 \pm 359 \mathrm{HU}$ and $3383 \pm 1234 \mathrm{HU}$ for high-dose scans. The differences between the window and level settings used for the low- and high-dose scans were statistically significant $(P<.05$, Mann-Whitney $U$ test) in both reviewer groups.

\section{Dosimetry}

Dosimetric data, including CTDI, DLP, and the effective doses of both the protocols used at our institution and literaturederived protocols, are shown in Table 2. Effective radiation doses of low-dose scans were approximately 6 times lower compared with those of high-dose scans and approximately 3-8 times lower compared with those of literature-derived protocols.

\section{Discussion}

The results of our study show that the normal temporal bone anatomy can be adequately assessed with low-dose CT in small children with a significant reduction in radiation exposure compared with the previously used high-dose protocol at our institution and among literature-derived protocols. ${ }^{8,9}$ Image quality is clearly reduced when using the low-dose protocol; however, the loss of image quality is reflected mainly by a shift from the subjective image-quality score of excellent to the scores of good and acceptable, while the frequency of insufficient scores was only nonsignificantly increased by using the low-dose protocol for neuroradiologist reviewers (Fig 1C). We, therefore, conclude that for neuroradiologists, the diagnostic image quality level is still maintained for low-dose scans despite the significant radiation dose reduction.

The diagnostic accuracy of our protocol for pathologies was not assessed directly. However, diagnostic work-up before middle ear surgery or cochlear implantation is possible with our protocol. First, findings of an otomastoiditis with fluid in the middle ear or mastoid, which are a concern in the preoperative work-up for any ear surgery, can still be reliably detected. Furthermore, the details of the cochlear anatomy that are important for inner ear surgery, like the modiolus and the cochlear patency, were still well-delineated on low-dose scans. The bony lamella between the internal auditory canal fundus and the cochlea may be helpful in the preoperative work-up for cochlear implantation because a bony dehiscence may be associated with a CSF gusher during the procedure. This structure is very thin and proved, indeed, to be marginal in lowdose scans; it was, however, rated as sufficiently visible in $93 \%$ and, hence, could be evaluated in most cases (Fig 2). Even if this structure is not assessable in some cases, the clinical relevance would be limited because an otologist is usually trained to solve an unexpected gusher intraoperatively and because the overall surgical strategy is not greatly influenced by a potential gusher. This potential shortcoming of low-dose protocols makes it, however, advisable to involve otologists, when such protocols are designed, so that they can have input on the degree of uncertainty that may remain.

Other pathologies may present a more challenging situation. Specifically, soft-tissue complications of mastoiditis, like intracranial or subperiosteal abscesses or sinus thrombosis, may not be adequately delineated due to the low softtissue resolution; hence, higher radiation doses are required for these purposes, particularly when intravenous contrast media are used.

As outlined above, we consider the image quality to be sufficient to delineate the anatomy (Fig 3). Nevertheless, the significantly higher fraction of insufficient ratings given by the otologists in the low-dose scans indicates that image quality is critical for small structures, like the vestibular and cochlear aqueduct, the bony lamella separating the cochlea from the internal auditory canal fundus, the modiolus, the oval window, the stapes, and the tympanic facial nerve segment. These structures must be reviewed with special attention when a low-dose protocol is used so as not to miss subtle abnormalities.

While no low-dose protocol for children has been described in the literature before, we found some temporal bone $\mathrm{CT}$ protocols in the literature for comparison (Table 2). The pediatric protocol (120 kV, $200 \mathrm{mAs})$ by Thomas and Wang," for example, results in 6-8 times higher effective doses compared with our protocol. In a widely used textbook ${ }^{8}$ on temporal bone imaging, the radiation dose issue and dose-reduction techniques for pediatric patients are discussed extensively. Age-adapted protocol recommendations are detailed for different age groups, including neonates, but the 

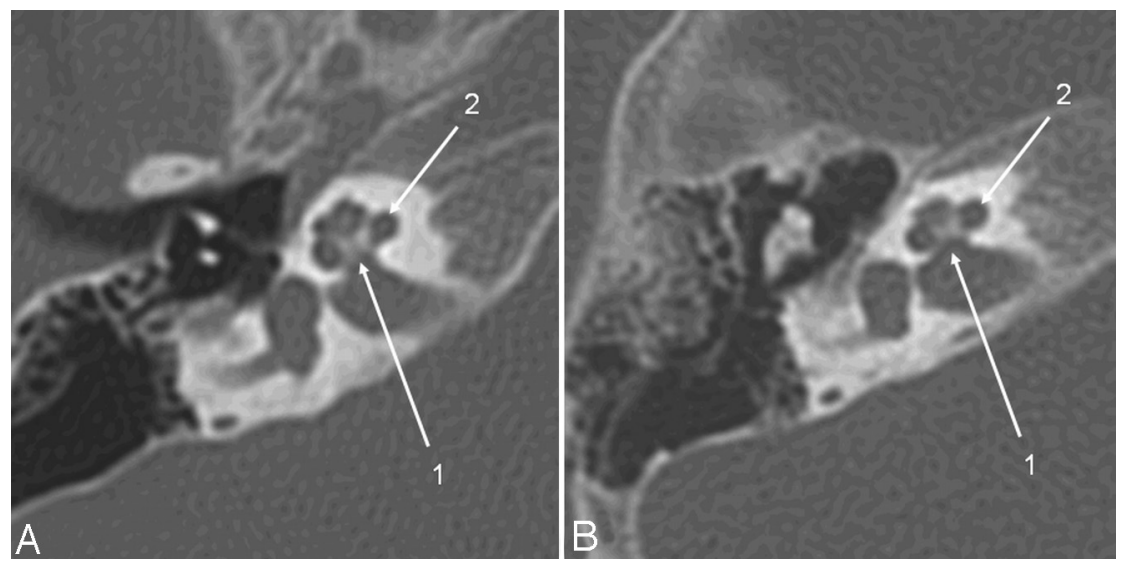

Fig 2. Axial CT section of the right temporal bone obtained with a CTDI vol $_{\text {of }} 63 \mathrm{mGy}$ (A) (14-month-old patient; DLP, 223 $\mathrm{mGy} \mathrm{cm}$; estimated $\mathrm{D}_{\text {eff }}, 1.4 \mathrm{mSv}$ ) and with the low-dose protocol (B) (16-month-old child; CTDI ${ }_{\text {vol, }} 10.8 \mathrm{mGy}$; DLP, 46.9 $\mathrm{mGy} \mathrm{cm}$; estimated $\mathrm{D}_{\text {eff }}, 0.35 \mathrm{mSv}$ ). Critical structures like the modiolus and the thin bony lamella separating the internal auditory canal from the cochlea (1) and the spiral osseous lamina (2) are delineated well despite the higher image noise.
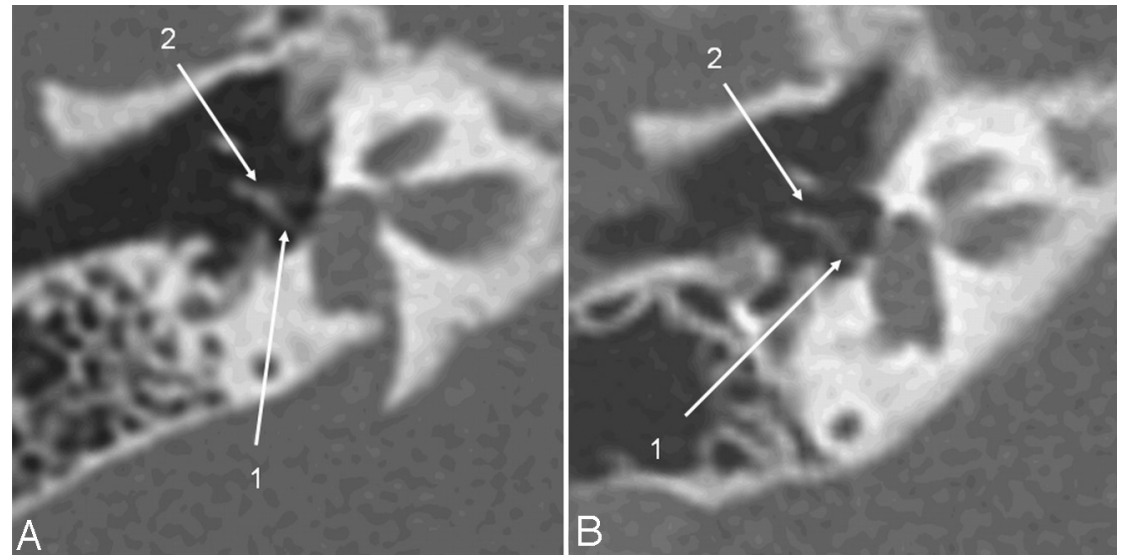

Fig 3. The same patients as in Fig 2. Oblique axial image reformatted in the stapes main plane, a high-dose scan $(A)$ versus a low-dose scan $(B)$. The posterior stapes crus (1) and the incudostapedial articulation (2) are identifiable on both scans.

Table 2: Mean effective doses (millisievert) for 3 age groups when scanned with local low- and high-dose protocols in comparison with literature-derived calculated effective doses and literature-derived effective doses

\begin{tabular}{|c|c|c|c|c|c|c|c|c|c|c|c|c|c|c|c|c|}
\hline \multirow[t]{2}{*}{ Age } & \multicolumn{3}{|c|}{ Low-Dose Protocol } & \multicolumn{3}{|c|}{ High-Dose Protocol } & \multicolumn{4}{|c|}{ Swartz et al $2009^{8 a}$} & \multicolumn{3}{|c|}{ Thomas et al $2008^{9 b}$} & \multicolumn{3}{|c|}{ Lutz et al $2007^{5 b}$} \\
\hline & CTDI & DLP & $D_{\text {eff }}$ & CTDI & DLP & $D_{\text {eff }}$ & CTDI & $\mathrm{kV}$ & $\mathrm{mAs}$ & $D_{\text {eff }}$ & $\mathrm{kV}$ & $\mathrm{mAs}$ & $D_{\text {eff }}$ & $\mathrm{kV}$ & mAs & $D_{\text {eff }}$ \\
\hline 1 year old & 8.8 & 40 & 0.3 & 63 & 285 & 1.8 & 34 & 120 & 150 & $0.9-1$ & 120 & 200 & 2.6 & - & - & - \\
\hline 5 year old & 10.8 & 49 & 0.25 & 63 & 288 & 1.4 & 45 & 120 & 200 & $0.97-1.1$ & 120 & 200 & 1.7 & - & - & - \\
\hline Adult & - & - & - & - & - & - & - & 120 & 320 & $1.2-1.3$ & - & - & - & 120 & 140 & 0.3 \\
\hline
\end{tabular}

a Indicates literature derived calculated effective doses

b - indicates literature-derived effective doses.

When available, CTDI (milligray), DLP (milligray $\times$ centimeter), and kilovolt/milliampere-second are shown also for literature-derived protocols.

settings, nevertheless, produce 3 times higher radiation doses compared with our protocol. The recommendations in the textbook likely strive for optimal image quality with justifiable radiation exposure, while our approach aims instead to reach the lowest radiation dose achievable as long as basic, yet diagnostic, image quality is maintained. For an adult temporal bone CT protocol, Lutz et $\mathrm{al}^{5}$ calculated effective doses that were similar to those resulting from our protocol.

However, when pediatric and adult effective doses are compared, an important point of consideration is that both the resulting effective dose from a particular set of scanner settings and the biologic impact of the effective dose differ between adults and children. ${ }^{2,10,11}$ The lifetime cancer mortality risk is estimated to be approximately $14 \%$ per sievert for a 1 -year-old child, $5 \%$ per sievert for a middle-aged adult, and $<2 \%$ per sievert for a person older than 60 years. ${ }^{12}$ Hence, even if the effective dose resulting from our protocol is comparable with the dose from an adult low-dose protocol, the associated stochastic risk is still approximately 2-3 times higher for an infant. This fact emphasizes the importance of using adequate settings during pediatric CT.

Considering our experiences, we propose the following procedure to obtain a low-dose temporal bone CT offering a sufficient image quality and advantageous effective doses: Start with $80 \mathrm{kV}$ and an empirically known CTDI ${ }_{\mathrm{vol}}$. Then, reduce the tube current stepwise as long as image quality remains sufficient. The structures that were the most difficult to visualize on low-dose scans were the stapes and the thin bony lamella separating the cochlea from the fundus of the internal auditory canal. It makes sense, therefore, to use these 2 structures as indicators of critical image quality when a low-dose protocol is implemented in practice. This approach should allow effective doses below $0.5 \mathrm{mSv}$ for young children.

On the basis of our results and data from literature, ${ }^{4}$ we further propose reviewing low-dose scans with broader window settings compared with high-dose scans because broader windows reduce the conspicuity of the image noise. 


\section{Limitations}

Our study has some limitations. First, image quality was only subjectively assessed by using a 5-point scale. No objective methods, such as measuring image noise or spatial resolution by using a high-contrast phantom, were applied. Despite this shortcoming, the use of subjective image quality scores is an accepted method and in widespread use in clinical radiology. ${ }^{5,13}$ In addition, the good interobserver variability of our readers validates the approach used in the study.

Another limitation, as previously outlined, is the fact that we did not assess the diagnostic accuracy of low-dose scans in pathologic temporal bone conditions. It would be difficult to validate the diagnostic accuracy of low-dose scans without the availability of high-dose scans of the identical patients for comparison. Such a study protocol would require a double radiation exposure and would be ethically difficult to justify. Instead, we recommend implementing stepwise dose-reduction protocols into clinical practice. The image quality obtained at each step should be assessed critically by reviewing several cases with different temporal bone pathologies before proceeding to the next lower dose, and otologists should be involved.

The inclusion criterion "normal anatomy" we used in our low-dose patient group leads to a selection bias because many infants with profound sensorineural hearing deficits do not show pathologic findings, as opposed to children scanned for infection or trauma. Furthermore, emergency studies, which were performed with a scanner from a different manufacturer located in the emergency department, were not included because image quality would not be comparable due to differences in scanner design and image reconstruction algorithms. These facts explain the high number of patients investigated for congenital hearing impairment in our study group; the distribution of indications in our cases does not reflect the whole spectrum of indications.

One might reason that CT of the temporal bone should be replaced by MR imaging because this approach would reduce the radiation exposure to zero. This approach may be justified for certain indications. However, there are still several reasons to use CT; a logistic reason may be the lack of MR imaging at an institution, and a more medical-based reason is the fact that temporal bone fractures or ossicular dislocations are not visualized on MR imaging. Another indication for CT is the preoperative assessment of cochlear implant candidates, in which the course of the facial nerve must be assessed and fibrous obliteration must be distinguished from cochlear ossification. $^{14}$ Furthermore, ossicular anomalies and traumatic changes cannot be evaluated by MR imaging. For the reasons mentioned here, we believe that there is still a need for pediatric temporal bone CT, and whenever children are exposed to ionizing radiation, it should be done with a dose-optimized protocol.

\section{Conclusions}

Low-dose temporal bone CT scans allow an accurate evaluation of middle and inner ear structures in infants and young children with radiation doses 3-8 times below literature-derived protocols with diagnostic, though reduced, image quality compared with that in high-dose scans. The image quality of small structures, such as the stapes or the bony lamella separating the cochlea from the fundus of the internal auditory canal, is, however, critical. These structures should be evaluated with special care on low-dose scans in order not to miss subtle pathologies. A close collaboration of neuroradiologists and otologists is therefore advised when low-dose temporal bone CT protocols are to be introduced into clinical routine.

\section{Acknowledgments}

We thank Mattheus Vischer and Jan Gralla, for valuable suggestions on the methodology, and Aisha Spring, photographer, for editing the figures.

\section{References}

1. Radiation Risks and Pediatric Computed Tomography (CT): A Guide for Health Care Providers. Rockville, Maryland: National Cancer Institute. 2008. Available at http//:www.cancer.gov/cancertopics/causes/radiation-risks-pediatric-CT. Accessed December 10, 2010

2. Vock P. CT dose reduction in children. Eur Radiol 2005;15:2330-40

3. Tack D. Methods and strategies for radiation dose optimization-and reduction-in MDCT with special focus on the image quality in computed tomography. In: Baert AL, Knauth M, Sartor K. Radiation Dose from Adult and Pediatric Multidetector CT. Berlin: Springer-Verlag; 2007:112

4. Husstedt HW, Prokop M, Dietrich B, et al. Low-dose high-resolution CT of the petrous bone. J Neuroradiol 2000;27:87-92

5. Lutz J, Jäger V, Hempel MJ, et al. Delineation of temporal bone anatomy: feasibility of low-dose 64-row CT in regard to image quality. Eur Radiol 2007;17:2638-45

6. Suetens P. Fundamentals of Medical Imaging. 2nd ed. Cambridge, United Kingdom: Cambridge University Press; 2009:59-63

7. Jones DG, Shrimpton PC. Normalised organ doses for $\mathbf{x}$-ray computed tomography calculated using Monte Carlo techniques. Chilton: National RadiologicalProtection Board.http://www.hpa.org.uk/web/HPAweb\&HPAwebStandard/ HPAweb_C/1195733753330. Accessed December 10, 2010

8. Swartz JD, Loevner LA. Imaging of the Temporal Bone. 4th ed. New York: Thieme; 2009:1-6

9. Thomas KE, Wang B. Age-specific effective doses for pediatric MSCT examinations at a large children's hospital using DLP conversion coefficients: a simple estimation method. Pediatr Radiol 2008;38:645-56

10. Huda W, Vance A. Patient radiation doses from adult and pediatric CT. AJR Am J Roentgenol 2007;188:540-46

11. Khursheed A, Hillier MC, Shrimpton PC, et al. Influence of patient age on normalized effective doses calculated for CT examinations. $\mathrm{Br} J$ Radiol 2002;75:819-30

12. Brenner DJ, Elliston CD, Hall EJ et al. Estimated risks of radiation-induced fatal cancer from pediatric CT. AJR Am J Roentgenol 2001;176:289-96

13. Singh S, Kalra MK, Moore MA, et al. Dose reduction and compliance with pediatric CT protocols adapted to patient size, clinical indication, and number of prior studies. Radiology 2009;252:200-08

14. Casselman JW. Diagnostic imaging in clinical neuro-otology. Curr Opin Neurol 2002;15:23-30 